\title{
Quadratic Weyl Representations
}

\author{
By
}

\author{
Paul L. ROBINSON*
}

\begin{abstract}
We offer a new construction for the quadratic representations of the canonical commutation relations introduced by Proksch, Reents and Summers; our construction, which is related to the metaplectic representation, automatically produces the quadratic representations in Weyl form, circumventıng the use of unbounded operators.
\end{abstract}

\section{Introduction}

Representations of the canonical commutation relations (CCR) feature prominently in quantum field theory, specifically in the description of bosonic systems. The underlying phase space for the CCR is fundamentally a real symplectic vector space: that is, a real vector space $V$ equipped with a symplectic (nondegenerate alternating real bilinear) form $\Omega$. A Heisenberg representation of the CCR assigns to each $v \in V$ a self-adjoint field operator $\Phi(v)$ in a fixed complex Hilbert space $\mathbb{H}$ satisfying the commutation relations

$$
x, y \in V \Rightarrow[\Phi(x), \Phi(y)] \subset i \Omega(x, y) I
$$

along with conditions designed to cope with the unboundedness of the field operators. A Weyl representation of the CCR assigns to each $v \in V$ a unitary Weyl operator $W(v)$ on a fixed complex Hilbert space $\mathbb{H}$ satisfying the commutation relations

$$
x, y \in V \Rightarrow W(x) W(y)=\exp \left\{\frac{1}{2 i \hbar} \Omega(x, y)\right\} W(x+y)
$$

together with a regularity condition amounting to strong continuity on finite-dimensional subspaces of $V$. Passage from a Weyl system to a Heisenberg

Communicated by T. Kawai, February 6, 1997.

1991 Mathematics Subject Classification : 81 S05

* Department of Mathematics, University of Florida, Gainesville FL 32611, U.S.A.

e-mail: plr@math.ufl.edu 
system is effected by associating to the strongly continuous one-parameter unitary group $(W(t \sqrt{\hbar} v): t \in \mathbb{R})$ its self-adjoint infinitesimal generator $\Phi(v)$ so that $W(t \sqrt{\hbar} v)=\exp \{i t \Phi(v)\}$.

When $V$ is a complex Hilbert space of whose complex inner product $\Omega$ is the imaginary part, there is a standard representation of the CCR over $V$ called the Fock representation, from which other representations of the CCR may be obtained by elementary transformations. Coherent transformations change the phase of the Weyl operators via multiplication by unitary characters of $V$. More substantially, quasifree transformations precompose the Weyl operators with symplectic automorphisms of $V$. These mechanisms for producing new representations of the $\mathrm{CCR}$ may be called (inhomogeneous) linear transformations.

In [4] appears a new class of representations of the CCR, called quadratic since they arise from the Fock representation by means of quadratic transformations. The approach in [4] calls for considerable technical skill, primarily because the authors construct the quadratic representations in Heisenberg form and verify that they exponentiate to yield Weyl representation. Our chief aim in this paper is to offer a fresh perspective on the quadratic representations by presenting them directly in explicit Weyl form, hence our title.

"Symplectic preliminaries" details some background real symplectic algebra of complex Hilbert spaces. "Weyl representations" is concerned largely with the Fock representations, for practical reasons in the version defined on $Q$-space; among other things, it is shown that symplectic automorphisms of $V$ acting identically on a Lagrangian subspace $L$ act canonically on the $Q$-space erected over $L$. "Quadratic representations" presents our explicit construction of the quadratic Weyl representations along with their transformation properties. Finally, we close with some brief remarks relating our construction to the one in [4].

\section{Symplectic Prelimimaries}

Let $V$ be a complex Hilbert space, whose complex inner product $\langle\cdot \mid \cdot\rangle$ has real part $\left(\cdot \bullet^{\circ}\right)$ and imaginary part $\Omega$. If $J: V \rightarrow V$ signifies multiplication by $i$ then

$$
x, y \in V \Rightarrow\langle x \mid y\rangle=\Omega(x, J y)+i \Omega(x, y) .
$$

The symplectic group $S p(V)$ comprises precisely all real-linear automorphisms $g$ of $V$ preserving the symplectic form $\Omega$ in the sense that

$$
x, y \in V \Rightarrow \Omega(g x, g y)=\Omega(x, y) \text {. }
$$


Note that if $g \in S p(V)$ then $g$ is necessarily bounded; its adjoint relative to $(\cdot \mid \cdot)$ is $g^{*}=-J g^{-1} J$. Our version of the symplectic Lie algebra $s p(V)$ comprises all (necessarily bounded) real-linear endomorphisms $\zeta$ of $V$ such that

$$
x, y \in V \Rightarrow \Omega(\zeta x, y)+\Omega(x, \zeta y)=0 .
$$

Of course, infinitesimal generators for arbitrary one-parameter subgroups of $S p(V)$ need not be bounded, so it is usual to define the symplectic Lie algebra differently; however, our needs are satisfied by bounded generators. Accordingly, the elementary exponential series defines the exponential map

$$
\exp : s p(V) \rightarrow S p(V) \text {. }
$$

Now, let $\Sigma: V \rightarrow V$ be a conjugation: thus, $\Sigma$ is antiunitary and $\sum \Sigma=I$. The real subspace $L \subset V$ of points fixed by $\sum$ is then a Lagrangian subspace and the $(\cdot \mid \cdot)$-orthogonal decomposition $V=J L \oplus L$ is a Lagrangian splitting, when $V$ is regarded as a real symplectic vector space. Denote by $S p(V)^{L}$ the group comprising precisely all $g \in S p(V)$ with the property that $g \mid L=I$ and by $s p(V)^{L}$ the Lie algebra of all $\zeta \in s p(V)$ with $\zeta \mid L=0$; of course, exp maps $s p(V)^{L}$ to $S p(V)^{L}$. In fact, we can describe $s p(V)^{L}$ quite intimately: specifically, in terms of the space $S^{L}$ comprising all (automatically bounded) real-linear endomorphisms $Z$ of $L$ that are self-adjoint in the sense

$$
x, y \in L \Rightarrow(Z x \mid y)=(x \mid Z y) \text {. }
$$

To begin, let $g \in S p(V)^{L}$. By definition, $g$ acts on $L$ as the identity operator. Let $h$ and $Z$ be the bounded real-linear endomorphisms of $L$ defined by

$$
z \in L \Rightarrow g J z=J h z+Z z .
$$

Now, let $x, y \in L$ : on the one hand,

$$
\begin{aligned}
\Omega(x, J y) & =\Omega(g x, g J y) \\
& =\Omega(x, J h y+Z y) \\
& =\Omega(x, J h y)
\end{aligned}
$$

whence $h=I$; on the other hand,

$$
\begin{aligned}
\Omega(x, y) & =\Omega(g J x, g J y) \\
& =\Omega(J x+Z x, J y+Z y) \\
& =\Omega(J x, J y)+\Omega(Z x, J y)+\Omega(J x, Z y) \\
& =\Omega(x, y)+(Z x \mid y)-(x \mid Z y)
\end{aligned}
$$

whence $Z \in S^{L}$. Thus we have a map

$$
S p(V)^{L} \rightarrow S^{L}: g \mapsto Z
$$

To continue, let $Z \in S^{L}$ and define a bounded real-linear endomorphism $\zeta$ of $V$ by 


$$
z, z^{\prime} \in L \Rightarrow \zeta\left(J z+z^{\prime}\right)=Z z
$$

so that $\zeta \mid L=0$ and if $x, x^{\prime}, y, y^{\prime} \in L$ then

$$
\Omega\left(\zeta\left(J x+x^{\prime}\right), J y+y^{\prime}\right)=\Omega(Z x, J y)=(Z x \mid y)
$$

whence $\zeta \in s p(V)^{L}$. Thus we have a map

$$
S^{L} \rightarrow s p(V)^{L}: Z \mapsto \zeta .
$$

To round out this discussion, we reconsider the exponential map. Note that if $\zeta \in s p(V)$ then $L \subset$ ker $\zeta$ exactly when $\operatorname{ran} \zeta \subset L$. It follows at once that if $\xi$, $\eta$ $\in s p(V)^{L}$ then their composite $\xi \eta: V \rightarrow V$ is zero, whence so is their bracket. Thus, $s p(V)^{L}$ is an abelian Lie algebra on which the exponential map assumes the very simple form

$$
\exp : s p(V)^{L} \rightarrow S p(V)^{L}: \zeta \mapsto I+\zeta
$$

and is actually a homomorphism of groups.

\section{Theorem $\mathbb{1}$.}

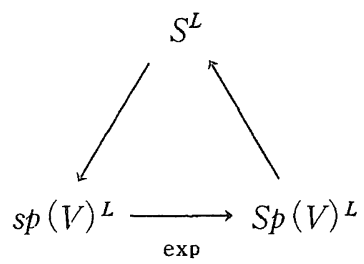

is a commutative triangle of isomorphisms between abelian groups.

Proof. Verification that three consecutive arrows yield the appropriate identity map is pleasantly routine.

It is this result that obviates the need for unbounded generators and permits us to focus on the bounded symplectic Lie algebra.

By definition, the restricted symplectic group $S p_{J}(V)$ is the subgroup of $S p(V)$ comprising precisely all $g \in S p(V)$ for which the antilinear part $A_{g}:=\frac{1}{2}$ $(g+J g J)$ is Hilbert-Schmidt, so that $g$ is the perturbation of its complex-linear part $C_{g}:=\frac{1}{2}(g-J g J)$ by a Hilbert-Schmidt operator. Analogously, we define $s p_{J}(V)$ to be the subalgebra of $s p(V)$ comprising all $\zeta \in s p(V)$ for which $A_{\zeta}$ is Hilbert-Schmidt. Finally, we define

$$
\begin{aligned}
& S p_{J}\left(V^{r}\right)^{L}=S p_{J}(V) \cap S p(V)^{L} \\
& s p_{J}(V)^{L}=s p_{J}(V) \cap s p(V)^{L} .
\end{aligned}
$$


Theorem 2. The exponential map defines an isomorphism of abelian gronps from $s p_{J}(V)^{L}$ to $S p_{J}(V)^{L}$.

Proof. This follows from Theorem 1 once it is recalled that if $\zeta \in s p(V)^{L}$ then $\exp \zeta=I+\zeta$ whence the antilinear parts of $\zeta$ and $\exp \zeta$ coincide.

Of course, the commutative triangle of isomorphisms in Theorem 1 induces one relating $s p_{J}(V)^{L}$ and $S p_{J}(V)^{L}$ to the space $S_{J}{ }^{L}$ comprising all HilbertSchmidt elements of $S^{L}$.

In fact, it is generally true that if $\zeta \in s p_{J}(V)$ then $\exp \zeta \in S p_{J}(V)$ : to see this, note that if $n \in \mathbb{N}$ and if $N(\zeta)$ denotes the Hilbert-Schmidt norm of the commutator $[J, \zeta]$ then inductively

$$
N\left(\zeta^{n}\right) \leq n\|\zeta\| n-1 N(\zeta)
$$

as a result of which the commutator

$$
[J, \exp \zeta]=\sum_{n=0}^{\infty} \frac{1}{n !}\left[J, \zeta^{n}\right]
$$

is a Hilbert-Schmidt operator.

We close this section with some structural clarification.

Theorem 3. $s p_{J}(V)^{L}$ comprises precisely all Hilbert-Schmidt operators in $s p$ $(V)^{L}$.

Proof. Recall that $\Sigma$ is the conjugation of $V$ having $L$ as its real space of fixed points. If $\zeta \in s p(V)^{L}$ then $\Sigma \zeta=\zeta$ so that

$$
\sum A_{5}=C_{5} \text {. }
$$

It follows that each of the separate conditions, that $A_{\zeta}$ be Hilbert-Schmidt and that $C_{\zeta}$ be Hilbert-Schmidt, is equivalent to the single condition that $\zeta=C_{\zeta}+A_{\zeta}$ itself be Hilbert-Schmidt.

Thus, $S p_{J}(V)^{L}$ comprises precisely all $g \in S p(V)^{L}$ for which $g-I$ is a Hilbert-Schmidt operator.

\section{Weyl Representations}

A regular Weyl representation of $V$ on a complex Hibert space $\mathbb{H}$ is a unitary projective representation $W: V \rightarrow$ Aut $\mathbf{H}$ with cocycle given by the rule

$$
x, y \in V \Rightarrow W(x) W(y)=\exp \left\{\frac{1}{2 i \hbar} \Omega(x, y)\right\} W(x+y)
$$


and with the (regularity) property that if $v \in V$ then the one-parameter unitary group $(W(t v): t \in \mathbb{R})$ is strongly continuous.

A specific irreducible regular Weyl representation of $V$ is its Fock representation, of which there are several standard models. As it will play a rôle in our subsequent discussion, we shall recall briefly the model known variously as the real wave representation or renormalized Schrödinger representation on $Q$-space; for further details, see [2] [3] [7] [8] [10].

To begin, we let $L \subset V$ be the real subspace fixed pointwise by the conjugation $\sum$ as before. Denote by $\mathscr{F}(L)$ the set of all finite-dimensional subspaces of $L$ directed under inclusion. Equip each $M \in \mathscr{F}(L)$ with the Gaussian (probability) measure $\mu$ having density function

$$
M \rightarrow \mathbb{R}: z \mapsto(\pi \hbar)^{-\frac{1}{2} \operatorname{dim} M} \exp \left(-\frac{(z \mid z)}{\hbar}\right)
$$

relative to normalized Lebesgue measure and write $P_{M}: L \rightarrow M$ for orthogonal projection. Let $H(M)$ stand for the set of all bounded complex functions $f: L \rightarrow$ $\mathbb{C}$ that are based on $M$ in the sense that $f=g \circ P_{M}$ for some bounded Borel function $g: M \rightarrow \mathbb{C}$. Elements of the complex vector space (indeed, algebra) $H(L)$ $:=\bigcup\{H(M): M \in \mathscr{F}(L)\}$ are called bounded tame functions. On occasion, we may lift the boundedness restriction and consider tame functions more generally.

Consistency of the Gaussian measures ensures that we may well define an inner product $\langle\cdot \mid \cdot\rangle$ on the complex vector space $H(L)$ of bounded tame functions by stipulating that if $f . g \in H(L)$ then

$$
\langle f \mid g\rangle=\int \bar{f} g d \mu
$$

where the integral extends over any $M$ in $\mathscr{F}(L)$ for which $f, g \in H(M)$. We shall denote the resulting Hilbert space completion of $H(L)$ by $\mathbb{H}(L)$ or simply $\mathbb{H}$ for convenience. Notice that if $M \in \mathscr{F}(L)$ then $\mathbb{H}(L)$ contains the Hilbert space completion $\mathbb{H}(M)$ of $H(M)$ and hence contains all tame functions $g \circ P_{M}$ with $g$ a polynomial. The Hilbert space $\mathbb{H}(L)$ is precisely $Q$-space.

The real wave representation $W$ of $V$ on $\mathbb{H}(L)$ arises as follows. For $v=J y$ $+x \in V$ with $x, y \in L$ we define $W(v)$ by requiring that

$$
[W(v) f](z)=\exp \frac{(x \mid 2 z-y)}{2 i \hbar} \exp \frac{(y \mid 2 z-y)}{2 \hbar} f(z-y) \text {. }
$$

This formula holds strictly for $z \in L$ and $f \in H(L)$ in which case $W(v) f$ is tame; of course, the unique extension to $\mathrm{H}_{\mathrm{H}}(L)$ is by continuity. We remark that our decision to fix $\frac{1}{2} \hbar$ as the variance of $\mu$ is not crucial; it has the virtue that the vacuum vectors for $W$ are simply the constant functions.

Henceforth, $W: V \rightarrow$ Aut $\mathrm{H}$ will be the real wave representation on $Q$-space 
as described above.

Plainly, if $g \in S p(V)$ then the composite $W \circ g: V \rightarrow$ Aut $\mathbf{H}$ is itself a regular Weyl representation. It is natural to ask whether $W \circ g$ is unitarily equivalent to $W$ in the sense that there exists a unitary operator $U \in$ Aut $\mathbf{H}$ with

$$
v \in V \Rightarrow W(g v)=U W(v) U^{-1} .
$$

Shale [9] answers this question: his answer may be expressed as the assertion that $W \circ g$ is unitarily equivalent to $W$ precisely when $g$ lies in the restricted symplectic group $S p_{J}(V)$. The group $M p_{J}^{c}(V)$ of all such intertwining unitary operators $U$ as $g$ runs over $S p_{J}(V)$ fits into a short exact sequence

$$
\mathbb{E}: 1 \rightarrow \mathbb{T} \rightarrow M p_{J}^{c}(V) \stackrel{\sigma}{\rightarrow} S p_{J}(V) \rightarrow 1
$$

where $\sigma(U)=g$ and $\mathbb{T}$ is the circle of unitary scalars. The tautologous unitary representation of $M p_{J}^{c}(V)$ on $\mathbb{H}$ is the metaplectic representation.

In the special case that $V$ is finite-dimensional, the group $M p_{J}^{c}(V)$ has a unique unitary character $\tau: M p_{J}^{c}(V) \rightarrow \mathbb{T}$ with the property that $\tau(\lambda)=\lambda^{2}$ when $\lambda \in \mathrm{T}$. The kernel of $\tau$ is a version of the connected double cover of $S p(V)$ known as the metaplectic group. In case $V$ is infinite-dimensional, the restricted symplectic group is actually contractible relative to a natural topology, so a direct analogue of $\tau$ cannot exist. Instead, an analogue of the metaplectic group twice covers the subgroup of $S_{p_{J}}(V)$ comprising all $g$ for which $C_{g}-I$ is trace class. See [6].

It is familiar that the short exact sequence $\mathbb{E}$ splits over the unitary group $U(V)$ of the complex Hilbert space $V$ : there is a homomorphism $U(V) \rightarrow M p_{J}^{c}(V)$ which yields the inclusion of $U(V)$ in $S p_{J}(V)$ when followed by $\sigma$; indeed, this is transparent in both the complex wave representation and the particle representation [2]. We may regard $U(V)$ as the subgroup of $S p(V)$ stabilizing a certain totally complex polarization of $V$ : namely, the subspace $\{v+i J v: v \in V\}$ of the complexification $V_{\mathbb{C}}$. Now, our fundamental Lagrangian subspace $L \subset V$ may also be regarded as a real polarization, of whose stabilizer $S p_{J}(V)^{L}$ is a distinguished subgroup. In fact, the short exact sequence $\mathbb{E}$ splits over $S p_{J}(V)^{L}$ : there exists a homomorphism $U: S p_{J}(V)^{L} \rightarrow M p_{J}^{c}(V)$ with the property that if $g$ $\in S p_{J}(V)^{L}$ then $\sigma\left(U_{g}\right)=g$. The remainder of this central section is devoted to establishing this important fact, a task to which the real wave representation is particularly well suited.

As a technical preliminary, we define a quadratic map

$$
\psi: L \rightarrow \mathbb{H}(L): z \mapsto \phi_{z}
$$

by 


$$
w \in L \Rightarrow \phi_{z}(w)=(z \mid w)^{2}-\frac{1}{2} \hbar(z \mid z)
$$

Theorem 4. If $x, y, z \in L$ then

$$
\begin{gathered}
\langle x \mid y\rangle=0 \Rightarrow\left\langle\phi_{x} \mid \phi_{y}\right\rangle=0 \\
\left\|\phi_{z}\right\|=\frac{\hbar}{\sqrt{2}}\|z\|^{2} .
\end{gathered}
$$

Proof. We shall assume $x, y, z$ to be unit vectors. Integrating over the plane spanned by $x$ and $y$ yields

$$
\begin{aligned}
\left\langle\phi_{x} \mid \phi_{y}\right\rangle & =\frac{1}{\pi \hbar} \iint\left(r^{2}-\frac{1}{2} \hbar\right)\left(s^{2}-\frac{1}{2} \hbar\right) e^{-\frac{r^{2}}{\hbar} e^{-\frac{s^{2}}{\hbar}} d r d s} \\
& =\left\{\frac{1}{\sqrt{\pi \hbar}} \int\left(t^{2}-\frac{1}{2} \hbar\right) e^{-\frac{t^{2}}{\hbar}} d t\right\}^{2}=0
\end{aligned}
$$

since $\mu$ has variance $\frac{1}{2} \hbar$. Higher moment calculations result in

$$
\left\|\phi_{z}\right\|^{2}=\frac{1}{\sqrt{\pi \hbar}} \int\left(t^{2}-\frac{1}{2} \hbar\right)^{2} e^{-\frac{t^{2}}{\hbar}} d t=\frac{1}{2} \hbar^{2}
$$

where integration is performed over the line spanned by $z$.

Similarly, if $z \in L$ then the homogeneous quadratic $\left(\left.z\right|^{\circ}\right)^{2}$ in $\mathbb{H}(L)$ satisfies

$$
\int(z \mid \cdot)^{2} d \mu=\frac{1}{2} \hbar\|z\|^{2}
$$

Now let $Z \in S_{J}^{L}$ be a Hilbert-Schmidt self-adjoint endomorphism of $L$. Let us agree to denote the Hilbert-Schmidt norm of $Z$ by $\|Z\|$ without further embellishment; no confusion will ensue, because no other operator norms will be written explicitly in the sequel.

Suppose first that $Z$ has finite rank: say $Z$ has range $M \in \mathscr{F}(L)$ having an orthonormal basis of eigenvectors $u_{1, \ldots, u_{m}}$ with corresponding eigenvalues $\lambda_{1}, \ldots, \lambda_{m}$ repeated according to their multiplicity. Thus,

$$
\|Z\|^{2}=\sum_{j=1}^{m} \lambda_{j}^{2}
$$

and

$$
z \in L \Rightarrow(z \mid Z z)=\sum_{j=1}^{m} \lambda_{j}\left(u_{j} \mid z\right)^{2}
$$

Also,

$$
\int(z \mid Z z) d \mu(z)=\sum_{j=1}^{m} \lambda_{i} \int(u, \mid \cdot)^{2} d \mu=\frac{1}{2} \hbar \operatorname{Tr} Z
$$


by the remark following Theorem 4 . We now define an inhomogeneous quadratic $\phi_{Z}: L \rightarrow \mathbb{R}$ by the prescription

$$
z \in L \Rightarrow \phi_{Z}(z)=(z \mid Z z)-\frac{1}{2} \hbar \operatorname{Tr} Z
$$

Theorem 5. If $Z \in S_{J}^{L}$ has finite rank then

$$
\left\|\phi_{Z}\right\|^{2}=\frac{1}{2} \hbar^{2}\|Z\|^{2}
$$

Proof. An immediate corollary to Theorem 4 in view of the decomposition

$$
\phi_{Z}=\sum_{j=1}^{m} \lambda_{j} \phi_{u,}
$$

Now lift the supposition of finite rank and let $Z \in S_{J}^{L}$ be arbitrary. When $M$ $\in \mathscr{F}(L)$ we shall denote by $Z_{M}$ the compression $P_{M} Z P_{M}$ of $Z$ so that $Z_{M} \in S_{J}^{L}$ is a finite rank operator to which the preceding considerations apply: when $Z$ is understood, we shall denote the inhomogeneous quadratic $\phi_{Z_{M}}$ simply by $\phi_{M}$ for convenience.

We shall use the following standard approximation result.

Theorem 6. The bounded net $\left(Z_{M}: M \in \mathscr{F}(L)\right)$ converges to $Z$ in $S_{J}^{L}$.

Proof. Explicitly, if $\varepsilon>0$ is given then there exists $M_{\varepsilon} \in \mathscr{F}(L)$ with the property

$$
M_{\varepsilon} \subset M \in \mathscr{F}(L) \Rightarrow\left\|Z-Z_{M}\right\| \leq \varepsilon .
$$

Briefly, let $Z$ have orthonormal eigenvectors $\left(u_{j}: j>0\right)$ with corresponding eigenvalues $\left(\lambda_{j}: j>0\right)$ so that $\|Z\|^{2}=\sum_{j>0} \lambda_{j}^{2}$ : if $N>0$ is chosen so large that $4 \sum_{j>N} \lambda_{j}^{2} \leq \varepsilon^{2}$ then $M_{\varepsilon}:=\operatorname{span}\left\{u_{1}, \ldots, u_{N}\right\}$ works; indeed, if $M_{\varepsilon} \subset M \in \mathscr{F}(L)$ then each of $\left\|Z-Z_{M \varepsilon}\right\|$ and $\left\|Z_{M \varepsilon}-Z_{M}\right\|$ is at most $\frac{1}{2} \varepsilon$.

We may now complete our present discussion of the inhomogeneous quadratics themselves: if $Z \in S_{J}^{L}$ is arbitrary then Theorem 5 and Theorem 6 together guarantee that the net $\left(\phi_{M}: M \in \mathscr{F}(L)\right)$ in $\mathbb{H}$ is Cauchy and therefore convergent to an element $\phi_{Z}$ of $\mathbb{H}$ satisfying

$$
\left\|\phi_{Z}\right\|^{2}=\frac{1}{2} \hbar^{2}\|Z\|^{2}
$$

Obviously, $\phi_{Z}$ has no hope for a pointwise formula as in the finite rank case unless $Z$ is trace class.

Again let $Z \in S_{J}^{L}$ be arbitrary and let $M \in \mathscr{F}(L)$. Multiplication by the 
unitary scalar function $\exp \left\{\frac{1}{2 i \hbar} \phi_{M}\right\}$ plainly defines an isometry $U_{M}: H(L) \rightarrow H$ $(L)$. Explicitly, if $f \in H(L)$ and $z \in L$ then

$$
\left[U_{M f}\right](z)=\exp \left\{\frac{1}{2 i \hbar} \phi_{M}(z)\right\} f(z) .
$$

If $K:=\sup |f|$ and if $M_{1}, M_{2} \in \mathscr{F}(L)$ then

$$
\left\|U_{M_{1}} f-U_{M_{2}} f\right\|^{2} \leq \frac{K^{2}}{4 \hbar^{2}} \int\left|\phi_{M_{1}}-\phi_{M_{2}}\right|^{2} d \mu
$$

by virtue of the elementary inequality

$$
t_{1}, t_{2} \in \mathbb{R} \Rightarrow\left|e^{i t_{1}}-e^{i t_{2}}\right| \leq\left|t_{1}-t_{2}\right| .
$$

Thus Theorem 5 yields

$$
\left\|U_{M_{1}} f-U_{M_{2}} f\right\| \leq \frac{K}{2 \sqrt{2}}\left\|Z_{M_{1}}-Z_{M_{2}}\right\|
$$

and so the net $\left(U_{M f}: M \in \mathscr{F}(L)\right)$ in $\mathbb{H}$ is Cauchy on account of Theorem 6 . The limit

$$
U f:=\lim _{M \rightarrow L} U_{M} f
$$

evidently satisfies the inequality

$$
M \in \mathscr{F}_{F}(L) \Rightarrow\left\|U f-U_{M} f\right\| \leq \frac{K}{2 \sqrt{2}}\left\|Z-Z_{M}\right\| .
$$

Continuous extension now uniquely defines an isometry $U: \mathbb{H} \rightarrow \mathbb{H}$. Of course, we may also uniquely extend each $U_{M}$ to an isometry of $\mathbb{H}$ and a standard $3 \varepsilon$ argument shows that the strong limit $U f=\lim _{M \rightarrow L} U_{M} f$ holds for every $f \in \mathbb{H}$.

Let us now formally indicate dependence on $Z \in S_{J}^{L}$ by writing $U_{Z}$ rather than $U$.

Theorem 7. If $X, Y \in S_{J}^{L}$ then

$$
U_{X} U_{Y}=U_{X+Y} .
$$

Proof. Write $Z=X+Y$ and for $M \in \mathscr{F}(L)$ let $U_{Z_{M}}$ be the isometry $U_{M}$ as above. Let $f \in \mathbb{H}$. For $\varepsilon>0$ choose $M_{\varepsilon} \in \mathscr{F}(L)$ so that if $M_{\varepsilon} \subset M \in \mathscr{F}(L)$ then both $\left\|U_{Y_{M}} f-U_{Y} f\right\| \leq \varepsilon$ and $\left\|U_{X_{M}}\left(U_{Y} f\right)-U_{X}\left(U_{Y} f\right)\right\| \leq \varepsilon$; as $U_{X_{M}}$ is an isometry, it follows that $\left\|U_{X_{M}} U_{Y_{M}} f-U_{X} U_{Y} f\right\| \leq 2 \varepsilon$ by the triangle inequality. The identity $U_{X_{M}} U_{Y_{M}} f=$ $U_{Z_{M}} f$ being plain, passage to limit as $M \rightarrow L$ yields $U_{X} U_{Y} f=U_{Z} f$ as required.

As $U_{0}$ is the identity operator, it follows at once that if $Z \in S_{J}^{L}$ then $U_{Z}$ is actually a unitary operator on $\mathbb{H}$. We remark that the homomorphism 


$$
U: S_{J}^{L} \rightarrow \text { Aut } \mathbf{H}: Z \mapsto U_{Z}
$$

has the property that each one-parameter group $\left(U_{t z}: t \in \mathbf{R}\right)$ is strongly continuous. For this, it is enough to verify continuity at zero: if $f \in H(L)$ and $\sup |f|=K$ then taking the limit $M \rightarrow L$ in

$$
\left\|U_{t Z_{M}} f-f\right\| \leq \frac{K}{2 \sqrt{2}}\left\|t Z_{M}\right\|
$$

yields

$$
\left\|U_{t Z} f-f\right\| \leq \frac{K}{2 \sqrt{2}}\|Z\||t|
$$

so that $\lim _{t \rightarrow 0} U_{t Z} f=f$; this result extends to $f \in \mathbf{H}$ by a standard $3 \varepsilon$ argument.

Finally, let $g \in \operatorname{Sp}_{J}(V)^{L}$ so that $Z:=(g-I) J \mid L \in S_{J}^{L}$ as usual and write $U_{g}$ in place of $U_{Z}$.

Theorem 8. If $g \in S p_{J}(V)^{L}$ then the unitary $U_{g} \in$ Aut $\mathbf{H}$ satisfies

$$
v \in V \Rightarrow W(g v)=U_{g} W(v) U_{g}^{-1} .
$$

Proof. It is enough to consider the cases $v=x$ and $v=J y$ for $x$ and $y$ in $L$. The unitary $W(x)$ acts on tame functions by multiplication, as does $U_{Z_{M}}$ whenever $M \in \mathscr{F}_{F}(L)$. Passage to the limit now shows that $U_{Z} W(x)=W(x) U_{Z}$ on H. Straightforward calculation verifies the equality $U_{Z_{M}} W(J y)=W(J y+Z y) U_{Z_{M}}$ on tame functions whenever $M \in \mathscr{F}(L)$. Passage to the limit shows that $U_{Z} W$ $(J y)=W(J y+Z y) U_{z}$ on $\mathbf{H}$ also.

We have thus constructed our splitting of the short exact sequence $\mathbf{E}$ over $S p_{J}(V)^{L}$. For future reference, we summarize the result as follows.

Theorem 9. There exists a homomorphism $U: S p_{J}(V)^{L} \rightarrow M p_{J}^{c}(V)$ such that if $g \in S p_{J}(V)^{L}$ then $\sigma\left(U_{g}\right)=g$ and if $\zeta \in s p_{J}(V)^{L}$ then the one-parameter unitary group $\left(U_{\exp t \zeta}: t \in \mathbb{R}\right)$ is strongly continuous.

To close this section, it is perhaps worth pointing out that the result of Shale [9] on unitary implementability of symplectic transformations was mentioned only to provide a context for our independent constructive argument leading to Theorem 9 .

\section{Quadratic Representations}

Our global construction of the quadratic Weyl representations introduced infinitesimally in [4] requires just one more algebraic ingredient. We let

$$
\zeta: V \rightarrow s p_{J}(V)^{L}: v \mapsto \zeta_{v}
$$


be a real-linear map possessing the symmetry property

$$
x, y \in V \Rightarrow \zeta_{x} y=\zeta_{y} x .
$$

We shall not assume of $\zeta$ any specific continuity properties, but note that it is automatically continuous when restricted to finite-dimensional subspaces of $V$. The commutative triangle of Theorem 1 associates to $\zeta$ a pair of group homomorphisms: the one we write as

$$
g: V \rightarrow S p_{J}(V)^{L}
$$

given by

$$
v \in V \Rightarrow g_{v}=\exp \left(\zeta_{v}\right)=I+\zeta_{v}
$$

and the other

$$
Z: V \rightarrow S_{J}^{L}: v \mapsto Z_{v}=\zeta_{u J} \mid L
$$

These homomorphisms have a number of special properties. Thus, just as elements of $s p_{J}(V)^{L}$ vanish on $L$ so does $\zeta: V \rightarrow s p_{J}(V)^{L}$ itself: indeed, if $z \in L$ and $v \in V$ then $\zeta_{z}(v)=\zeta_{v}(z)=0$. We shall have need of the following rather peculiar properties.

Theorem 10. If $x, y \in V$ then

$$
g_{x+y}(x+y)=g_{x} x+g_{x}^{2} g_{y} y
$$

and

$$
\begin{gathered}
\Omega\left(x+y, g_{x+y}(x+y)\right)= \\
\Omega\left(x, g_{x} x\right)+\Omega\left(y, g_{y} y\right)+3 \Omega\left(x, \zeta_{x+y} y\right) .
\end{gathered}
$$

Proof. The first identity comes from simple expansion using the symmetry of $\zeta$. The second comes from the first by expansion, using symmetry of $\zeta$ and the fact that if $\xi, \eta \in s_{J}(V)^{L}$ then $\xi \eta=0$.

Our reason for drawing attention to the second property in Theorem 10 is that the map $\chi: V \rightarrow \mathbb{T}$ defined by the prescription that if $v \in V$ then

$$
\chi(v)=\exp \left\{\frac{1}{6 i \hbar} \Omega\left(v, \zeta_{v} v\right)\right\}=\exp \left\{\frac{1}{6 i \hbar} \Omega\left(v, g_{v} v\right)\right\}
$$

has the property that if $x, y \in V$ then

$$
\chi(x+y)=\chi(x) \chi(y) \exp \left\{\frac{1}{2 i \hbar} \Omega\left(x, \zeta_{x+y} y\right)\right\}
$$

so

$$
\exp \left\{\frac{1}{2 i \hbar} \Omega(x, y)\right\} \chi(x+y)=\chi(x) \chi(y) \exp \left\{\frac{1}{2 i \hbar} \Omega\left(g_{x} x, g_{x}^{2} g_{y} y\right)\right\}
$$


Our reason for drawing attention to this and the first property in Theorem 10 will soon be clear.

Following $g: V \rightarrow S p_{J}(V)^{L}$ by the splitting $S p_{J}(V)^{L} \rightarrow M p_{J}^{c}\left(I^{r}\right)$ of $\sigma$ in Theorem 9 provides a homomorphism which we shall denote abusively by

$$
U: V \rightarrow M p_{J}^{c}(V): v \mapsto U_{v}
$$

so that

$$
w \in V \Rightarrow W\left(g_{v} w\right)=U_{v} W(w) U_{v}^{-1}
$$

and the one-parameter group $\left(U_{t v}: t \in \mathbb{R}\right)$ is strongly continuous.

Having made all of these preparations, we now define a map

$$
W_{\zeta}: V \rightarrow \text { Aut } \mathbb{H}
$$

by the rule that if $v \in V$ then

$$
W_{\zeta}(v)=\chi(v) L_{v} W(v) U_{v}
$$

or equivalently

$$
W_{\zeta}\left(v^{\prime}\right)=\chi\left(v^{\prime}\right) W\left(g_{v} v^{\prime}\right) U_{v}^{2}
$$

Theorem 11. $W_{\zeta}$ is a regular Weyl representation.

Proof. To confirm that $W_{\xi}$ has the correct cocycle, note that if $x, y \in V$ then

$$
\begin{aligned}
W_{\zeta}(x) W_{r}(y) & =\chi(x) \chi(y) W\left(g_{x} x\right) U_{x}^{2} W\left(g_{y} y\right) U_{y}^{2} \\
& =\chi(x) \chi(y) W\left(g_{x} x\right) W\left(g_{x}^{2} g_{y} y\right) U_{x}^{2} U_{y}^{2} \\
& =\chi(x) \chi(y) \exp \left\{\frac{1}{2 i \hbar} \Omega\left(g_{x} x, g_{x}^{2} g_{y} y\right)\right\} W\left(g_{x} x+g_{x}^{2} g_{y} y\right) U_{x+y}^{2} \\
& =\exp \left\{\frac{1}{2 i \hbar} \Omega(x, y)\right\} \chi(x+y) W\left(g_{x+y}(x+y)\right) L_{x+y}^{2} \\
& =\exp \left\{\frac{1}{2 i \hbar} \Omega(x, y)\right\} W_{\zeta}(x+y)
\end{aligned}
$$

on account of Theorem 10 and the subsequent remark. Regularity is plain. for if $v \in I^{r}$ then each of the unitaries $W\left(t v^{\prime}\right)$ and $L_{t v}$ is strongly continuous in $t \in \mathbb{R}$.

We refer to $W_{\zeta}: V \rightarrow$ Aut $\mathbb{H}$ so constructed as the quadratic Weyl representation associated to the symmetric linear map $\zeta: V \rightarrow s p_{J}(V)^{L}$.

Notice that there is a little freedom in our construction: the homomorphism $U^{U}: V^{\gamma} \rightarrow M p_{J}^{c}(V)$ may be multiplied by a unitary character $\kappa=\exp \circ\left\{\frac{1}{21 \hbar} \lambda\right\}$ where $\lambda: V \rightarrow \mathbb{R}$ is a real-linear map; the quadratic Weyl representation is then 
multiplied by $\kappa^{2}=\exp \circ\left\{\frac{1}{\hbar} \lambda\right\}$. Of course, this amounts to the freedom of a coherent transformation.

A symplectic transformation offers more entertainment, touching upon functoriality of the real wave representation. For this, let $a \in S p(V)$ be arbitrary. This transformation provides $V$ with a new structure of complex Hilbert space, having complex structure $J^{\prime}=a J a^{-1}$ and complex inner product $\langle\cdot \mid \cdot\rangle^{\prime}$ given by

$$
x, y \in V \Rightarrow\langle x \mid y\rangle^{\prime}=\Omega\left(x, J^{\prime} y\right)+i \Omega(x, y)
$$

with the property that

$$
x, y \in V \Rightarrow\langle a x \mid a y\rangle^{\prime}=\langle x \mid y\rangle .
$$

Naturally, the real part $(\cdot \mid \cdot)^{\prime}$ of $\langle\cdot \mid \cdot\rangle^{\prime}$ behaves in a similar fashion. The conjugation $\Sigma^{\prime}:=a \sum a^{-1}$ has the Lagrangian $L^{\prime}:=a L$ as its space of fixed points. Upon $L^{\prime}$ with $(\cdot /)^{\prime}$ as inner product we erect the version $\mathbb{H}^{\prime}:=\mathbb{H}\left(L^{\prime}\right)$ of $Q$-space. We also define the version $W^{\prime}: V \rightarrow$ Aut $\mathbb{H}^{\prime}$ of the real wave representation by continuously extending the rule

$$
\begin{aligned}
& {\left[W^{\prime}\left(J^{\prime} y^{\prime}+x^{\prime}\right) f^{\prime}\right]\left(z^{\prime}\right)=} \\
& \quad \exp \frac{1}{2 i \hbar}\left\{\left(x^{\prime} \mid 2 z^{\prime}-y^{\prime}\right)^{\prime}+i\left(y^{\prime} \mid 2 z^{\prime}-y^{\prime}\right)^{\prime}\right\} f\left(z^{\prime}-y^{\prime}\right)
\end{aligned}
$$

for $x^{\prime}, y^{\prime}, z^{\prime} \in L^{\prime}$ and $f^{\prime} \in H\left(L^{\prime}\right)$. It is readily confirmed that continuous extension of the isometry

$$
A: H(L) \rightarrow H\left(L^{\prime}\right): f \mapsto f \circ a^{-1}
$$

yields a unitary isomorphism $A: \mathbb{H} \rightarrow \mathbb{H}^{\prime}$ having the property

$$
v \in V \Rightarrow W^{\prime}(a v)=A W(v) A^{-1} .
$$

This is functoriality of the real wave representation. A readily confirmed aspect of this functoriality is the fact that the splitting homomorphism $U^{\prime}: S p_{J^{\prime}}(V)^{L^{\prime}} \rightarrow$ $M p_{J^{\prime}}^{c}(V)$ constructed parallel to Theorem 9 satisfies

$$
g \in S p_{J}(V)^{L} \Rightarrow U^{\prime}\left(a g a^{-1}\right)=A U(g) A^{-1} .
$$

Now $a \in S p(V)$ acts naturally on the symmetric linear map $\zeta: V \rightarrow s p_{J}(V)^{L}$ to produce $\zeta^{\prime}: V \rightarrow s p_{J^{\prime}}(V)^{L^{\prime}}$ given by

$$
v \in V \Rightarrow \zeta_{a v}^{\prime}=a \zeta_{v} a^{-1}
$$

and the map $\chi^{\prime}: V \rightarrow \mathbb{T}$ given by

$$
v \in V \Rightarrow \chi^{\prime}(v)=\exp \left\{\frac{1}{6 i \hbar} \Omega\left(v, \zeta_{v}^{\prime} v\right)\right\}
$$


satisfies $\chi^{\prime}=\chi \circ a^{-1}$. In terms of established notation, we now have the following result.

Theorem 12. If $v \in V$ then

$$
W_{\zeta^{\prime}}^{\prime}(a v)=A W_{\zeta}(v) A^{-1}
$$

Proof. A matter of direct calculation. If $g^{\prime}:=\exp \circ \zeta^{\prime}$ then

$$
\begin{aligned}
W_{\zeta^{\prime}}^{\prime}(a v) & =\chi^{\prime}(a v) U^{\prime}\left(g_{a v}^{\prime}\right) W^{\prime}(a v) U^{\prime}\left(g_{a v}^{\prime}\right) \\
& =\chi(v) U^{\prime}\left(a g_{v} a^{-1}\right) A W(v) A^{-1} U^{\prime}\left(a g_{v} a^{-1}\right) \\
& =A\left\{\chi(v) U\left(g_{v}\right) W(v) U\left(g_{v}\right)\right\} A^{-1} \\
& =A W_{\zeta}(v) A^{-1} .
\end{aligned}
$$

With this symplectic functoriality of the quadratic Weyl representations we close the present section.

\section{Remarks}

At this point, it is appropriate to relate our construction to the one in [4].

The construction in [4] involves a (without essential loss) maximal subspace $L \subset V$ on which $\Omega$ is identically zero and assumes a real-linear map $\Lambda$ $: V \rightarrow L \otimes L \subset V \otimes V$ with the property that if $x, y \in V$ then $\Lambda_{x}(y)=\Lambda_{y}(x)$ where elements of $V \otimes V$ act on $V$ by linear extension of the rule that if $x, y, z \in V$ then $(x \otimes y) \cdot z=\Omega(x, z) y$. It is straightforward to verify that if $v^{\prime} \in V$ then $\Lambda_{v} \in L \otimes L$ corresponds to our $Z_{v} \in S_{J}^{L}$ under the natural identification of the real tensor product $L \otimes L$ with the space $S_{J}^{L}$ of Hilbert-Schmidt self-adjoint endomorphisms of $L$.

The construction in [4] is also based on a rule $\Psi$ for passing from (without loss, symmetric) elements of the real tensor product $V \otimes V$ to essentially self-adjoint operators in Fock space, these operators being bilinear Hamiltonians in the sense of Araki [1]. In fact, $\Psi$ actually constructs infinitesimal generators for the metaplectic representation; in hindsight, this is clear from the commutation relations in [4] Proposition 3.2.6. The Wick ordering of $\Psi$ in [4] is manifest in our rule $Z \mapsto \phi_{Z}$ for the construction of inhomogeneous quadratics.

With $\Lambda$ and $\Psi$ as above, the authors of [4] set $\hbar=1$ and for $v \in V$ define a transformed field operator $\Phi_{\Lambda}(v)$ to be the closure of the essentially self-adjoint operator $\Phi(v)+\Psi(\Lambda v)$ where $\Phi(v)$ is the Fock field operator satisfying $\exp \{i \Phi$ $(v)\}=W(v)$. That the transformed field operators satisfy the Heisenberg form 
of the canonical commutation relations

$$
x, y \in V \Rightarrow\left[\Phi_{\Lambda}(x), \Phi_{\Lambda}(y)\right] \subset i \Omega(x, y) I
$$

rests partly on the fact that what is effectively the infinitesimal metaplectic cocycle ( $\sigma$ in [4] Proposition 3.2.6) vanishes on the range of $\Lambda$. This vanishing is a consequence of our Theorem 9 which asserts the triviality over $S p_{J}(V)^{L}$ of the global metaplectic cocycle. With the preceding identifications, our $W_{\zeta}$ of course reproduces the representation $W_{A}$ defined in [4] by $W_{\Lambda}(v)=\exp \left\{i \bar{\Phi}_{\Lambda}\right.$ $\left.\left(\imath^{\prime}\right)\right\}$ for $v^{\prime} \in V$.

Our construction offers a fresh perspective on the quadratic Weyl representations. For example, it facilitates an alternative approach to the problem of determining necessary and sufficient conditions for $W_{\zeta}$ to be unitarily equivalent to the original Fock representation, a problem settled in [4]. In fact, it enables an approach to the more general problem of determining necessary and sufficient conditions for $W_{\zeta}$ to be unitarily equivalent to any Fock representation whatsoever, a problem on which we plan to report elsewhere. We also plan to determine the extent to which the unitary equivalence class of $W_{\zeta}$ depends on $\zeta$.

Finally, in the last chapter of [4] the question is raised whether it is possible to construct representations of the canonical commutation relations having order higher than quadratic, the comment being made that verification of (essential) self-adjointness for the pertinent field operators is likely to be difficult. Being global rather than infinitesimal, our construction of the quadratic representations avoids such problems of self-adjointness. This suggests that a search for higher order representations along similar lines might prove fruitful.

\section{References}

[1] Arakı, H.. On Quasifree States of the Canonical Commutation Relations (II), Puibl. RIMS. Kryoto Unv?, 7(1971/72). 121-152

[2] Baez, J. C. Segal. I. E. and Zhou, Z., Introduction to Algebranc and Constructive Quantum Field Theory. Princeton University Press. 1992.

[3] Glimm. J. and Jaffe. A., Boson Quantum Field Modcls, in Mathematus of Contemporary Physics, Academic Press (1972), 77-143.

[4] Proksch, M., Reents, G. and Summers. S J. Quadratic Representations of the Canonical Commutation Relations, Publ RIMS. Kyoto (intu' 31 (1995), 755-804.

[5] Reents. G and Summers. S J. Bevond coherent states higher order representations, in On Klauder's Path \& Field Trlp. World Scientific, Singapore (1994), 179-188.

[6] Robinson. P. L.. An infinite-dimensional metaplectic group. Quart. J. Math. Oxford, 43 (1992). 243-252.

[7] Segal. I E.. Tensor algebras over Hilbert spaces I. Trans. Amer. Math. Soc., 81 (1956), 106-134.

[8] - Mathematıcal Problems of Relatın'stıc Physics, American Mathematical Society 1963 
[9] Shale. D.. Linear symmetries of free boson fields. Trans. Amer. Math. Soc., 103 (1962), 149-167.

[10] Simon, B., The $P(\phi)_{2}$ Eucludean (Quantum) Field Theorv. Princeton University Press, 1974. 
